http://dx.doi.org/10.21611/qirt.1992.020

\title{
Infrared thermography of plastic instabilities in a single crystal superalloy
}

\author{
by P. LEVESQUE $\left({ }^{\star}\right)$, B. LISIECKI $\left(^{\star \star}\right)$, L. KUBIN $\left(^{* \star}\right)$, \\ P. CARON $\left({ }^{* *}\right)$, A. DEOM $(*)$ and D. BALAGEAS $\left(^{*}\right)$
}

(*) ONERA , (**) LEM, CNRS/ONERA, BP 72 ,F-92322 Châtillon cedex, France.

\begin{abstract}
A tensile test of a AM3 single crystal superalloy is analysed with the help of the thermographic technique. During the plastic flow, with a moderate strain rate at room temperature, the deformation is very localized and large slip bands occur on the surface of the specimen, and the stress-strain curve exhibits yielding serrations. The IR thermography is used to measure local heatings. The heating associated with the deformation band occurrence, during the instability, is evaluated by a time-resolved analysis of the thermograms delivered by the IR camera, using a very simple thermal model. A value of about $140^{\circ}$ is found and compared to the upper possible limit of this parameter, given by theoretical calculations and analysis of the tensile curve.
\end{abstract}

\section{Nomenclature}

C volume heat capacity $\left(\mathrm{J} \mathrm{m}^{-3} \mathrm{~K}^{-1}\right)$

$D$ diameter of the specimen

$M$ combined elastic modulus

$\Delta T$ measured specimen heating $(K)$

$\Delta T_{0}$ initial wall temperature $(K)$

$V_{0}$ volume of the specimen

$\alpha$ constant assumed to be 0.9

$\sigma_{0}$ stress at which the stress drop occurs

$2 a \quad$ width of the slip band

$t$ time (s)

$x \quad$ distance from the slip band to the

monitored point

vo volume of the slip band

$\kappa \quad$ thermal diffusivity $\left(m^{2} s^{-1}\right)$

$\Delta \sigma \quad$ stress drop

\section{Introduction}

Single crystalline $\gamma / \gamma^{\prime}$ nickel based superalloys are used in aeronautical gas turbine engines as blade and vane materials. The AM3 superalloy, recently developed at ONERA [1], shows a very good specific strength due to its relatively low density $(d=8.25)$. The tensile behaviour of these single crystalline materials is characterized by plastic instabilities occurring at yielding. Despite the fact that these features were reported for many superalloys no specific study was devoted to the description and the understanding of this particular behaviour.

During the plastic deformation, about $85 \%$ of the injected energy in the specimen is dissipated as heat and the remainder is stored in the material in the form of dislocation energy. For conventional strain rates and for homogeneous deformations, the rise in temperature is too low to be detected. In some unusual cases, for instance with high strain rate, tests at cryogenic temperatures or when the plastic deformation is heterogeneous, the rise in temperature can be high. The last case corresponds to plastic instabilities. Each instability corresponds to the sudden formation of one or several slip bands parallel to two slip planes \{111\}. Scanning electronic microscopy and transmission electronic microscopy observations show that the $\gamma$ precipitates are sheared or rotated by the motion of dislocations during localized events. Each instability is accompanied by a sound burst which indicates that the dislocations move at near the sound velocity. An instability lasts about $\delta t \approx D / v$ ( $D$ being the diameter of the specimen and $v$ the average dislocation velocity in the slip band). Within this duration, the dislocations move 


\section{http://dx.doi.org/10.21611/qirt.1992.020}

on a distance $D$, ranging about the diameter of the specimen. With $v \approx 10^{2}-10^{3} \mathrm{~m} . \mathrm{s}^{-1}$ and $D=3 \mathrm{~mm}, \delta t \approx 3$ to $30 \mu \mathrm{s}$. On the other hand, the shearing is about $10^{-2}$ in the deformation bands and the local strain rate can then reach $10^{3}-10^{4} \mathrm{~s}^{-1}$.

It seemed interesting to estimate the local temperature increase in the AM3 superalloy during the plastic instabilities. A first approach as been carried out in measuring on the tensile curve the injected energy and converting it into heat with an adiabatic assumption. Although this assumption is justified by the very short duration of the phenomena (see below), a more direct measurement of the heating was wanted. Because the classical infrared thermography has no access to such fast events, a particular method which allows a back-extrapolation of the initial temperature burst was to be developed.

\section{Specimen and test conditions}

The nominal composition of the AM3 alloy is $6 \mathrm{Al}, 2 \mathrm{Ti}, 3.5 \mathrm{Ta}, 5 \mathrm{~W}, 2.25 \mathrm{Mo}, 8 \mathrm{Cr}, 5.5 \mathrm{Co}$, balance $\mathrm{Ni}$, in weight percent. Single crystal rods of $\mathrm{AM} 3$ were cast by the withdrawal process using seed crystals oriented along [011]. The crystals orientations were determined by the Laüe back reflection $X$ ray technique. The mechanical properties were studied after the following heat treatment. The heat treatment, $1315^{\circ} \mathrm{C}$ for $3 \mathrm{~h}$ followed by air cooling, results in a irregular distribution of odd shaped $\gamma^{\prime}$ particules, about $0.3 \mu \mathrm{m}$ in size, with a volume fraction of $70 \%$.

Cylindrical tensile specimens were machined from the rods with a diameter of $3 \mathrm{~mm}$ and a gauge length of $25 \mathrm{~mm}$. Tensile tests were performed on a Schenck testing machine with a constant cross head speed $(0.2 \mathrm{~mm} / \mathrm{min})$, corresponding to an initial strain rate of $1.4 \times 10^{-4} \mathrm{~s}^{-1}$.

\section{Infrared thermographic measurements}

\subsection{Experimental procedures}

During the tensile tests at room temperature, infrared thermography has been used to detect the local heat emission occuring during the plastic instabilities. The infrared thermographic system consists in an AGEMA thermovision 782 LW camera associated with the Cedip PTR-9000 system for data acquisition, digitization and storage. The time definition is 25 frames per second with 100 lines per frame, each line being composed of 125 points. In fact, among these 100 lines, only 64 are actually used to construct the image; so the image is constructed by interlacing a sequence a four successive frames. The time definition is therefore $40 \mathrm{~ms}$, much lower than the assumed duration of an instability. When the vertical scanning is suppressed, the thermal field can be explored along one line, at a frequency of $2,500 \mathrm{~Hz}$. The detection is sensitive to wavelengths in the range $8-12 \mu \mathrm{m}$ and converts the IR signal into a thermal signal whose amplitude varies with time according to the specimen luminance. The thermal signal is then processed numerically, yielding a thermal image. To minimize the reflection of radiation coming from the environment, an opaque coating of black paint with an emission of $85 \%$ was sprayed on the surface.

\subsection{Experimental results}

Infrared thermography allows to visualize the occurrence of the deformation bands during the tensile test. Figure $A^{*}$ presents a thermal image showing the simultaneous development of two deformation bands along two different slip systems during an instability. Figure 1 is the corresponding SEM image, showing that one $\{111\}<110>$ slip system is distributed rather homogeneously while a second appears in the form of a single slip line.

A series of infrared images taken by the camera, allowing the detection of an instability and the progress of the diffusioni of the energy generated in the slip band is given in figure 3 . The configuration being given in figure 2 . Figure 3 shows a sequence of thermal events, where the time origin is the start of the acquisition. Frame 1 taken at time $t=2.92 \mathrm{~s}$, shows no evidence of thermal burst. In the time range $2.92-2.96 \mathrm{~s}$, an instability occurs, because a heat band appears

\footnotetext{
* The colour plates of this article 20 are located on page $V /$ of the colour gathering, at the end of the book
} 




Fig.1. - Deformation bands in two different slip systems (one appears in the form of a single slip line) seen by SEM. This image is to be compared to the infrared image of Fig. A.

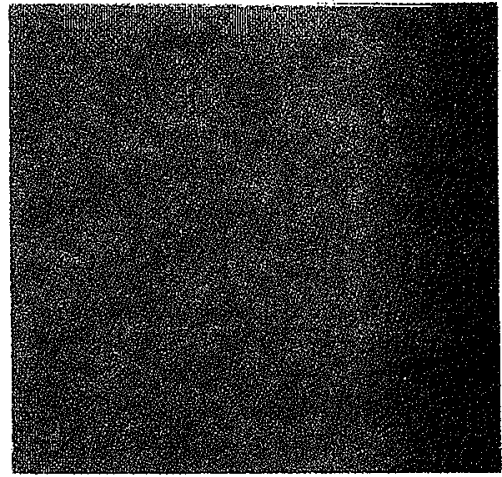

frame $1: t=2.92 \mathrm{~s}$

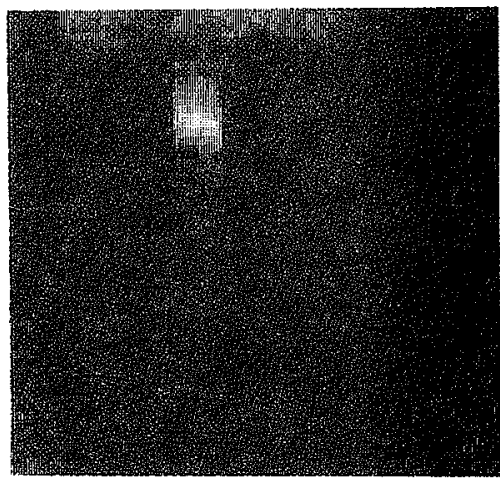

frame $3: t=3.00 \mathrm{~s}$

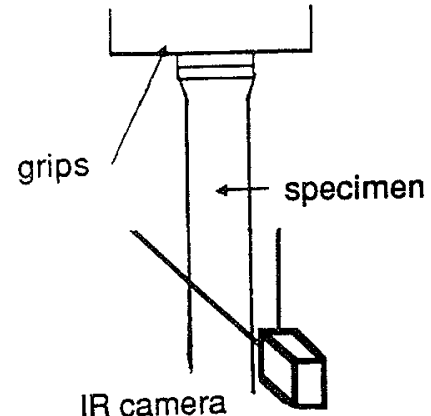

Fig.2.- Experimental configuration and fleld of view of the camera with the $7^{\circ} \times 7^{\circ}$ objective

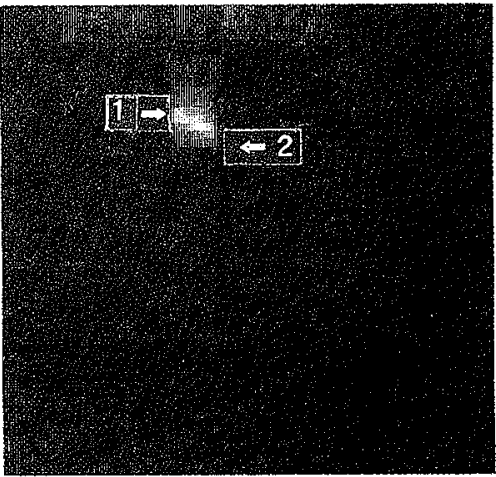

frame $2: t=2.96 \mathrm{~s}$

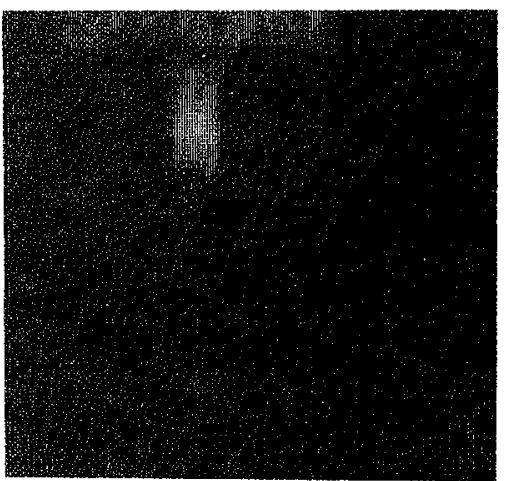

frame $10: t=3.28 \mathrm{~s}$

Fig. 3. - Detection by the infrared camera of an instability which occurs between frame times of $2.92 \mathrm{~s}$ and $2.96 \mathrm{~s}$ and diffusion along the specimen axis of the generated heat from the slip band into the specimen. The unheated part of the specimen is hardly visible since at ambient temperature (see schematic arrangement in figure 2). 
on the frame 2 , taken at $t=2.96 \mathrm{~s}$. On the following frames, we observe a decrease in temperature as heat diffuses along the axis of the specimen. From these thermal images two points (pixels), located on the specimen axis, have been selected and their evolution with time was investigated in detail. Point 1, which is the hottest, is located very near of the slip band, and point 2 is at a distance of $1.3 \mathrm{~mm}$ from it. For these two points, the temperature time evolution is given in figure 4. The thermogram of point 1 exhibits a sharp peak corresponding to the arrival of the heat burst generated very near, followed by a slower decrease due to the diffusion of heat in the specimen. For point 2 , the thermal peak is lower and later, because the point is farther off the source.

SEM observation, after tensile test, has allowed to identify the deformation band and to measure its width, which is about $38 \mu \mathrm{m}$.

\subsection{Determination of the Initial temperature rise in the slip band}

The experimental results presented below are to be interpreted to determine the initial temperature rise within the slip band, $\Delta T_{0}$. The thermal problem can be stated as follows : at time $t=0$ the initial temperature distribution is zero in the whole sample, the slip band excepted. In this region, whose width is $2 a$, the temperature is $\Delta T_{0}$. The one-dimension solution of the heat diffusion equation is applicable since the specimen can be considered as an infinite medium if no heat losses are assumed at its surface. The solution is:

$$
\Delta T(t)=\Delta T_{o}\left[e r f\left[(a-x) /(4 \kappa t)^{1 / 2}\right]+\operatorname{erf}\left[(a+x) /(4 \kappa t)^{1 / 2}\right]\right] / 2
$$

where $\mathrm{k}$ is the diffusivity of the single crystal AM3, $x$ the distance from the monitored point to the middle of the band and $\operatorname{erf}(u)$ is the error function. The only unknown, $\Delta T_{0}$, is identified by fitting the experimental thermogram, in its peak region, with expression (1) where the following values are assumed: $x=1.3 \mathrm{~mm}, 2 a=38 \mu \mathrm{m}$ and $x=2.410^{-6} \mathrm{~m} .{ }^{2} \mathrm{~s}^{-1}$. The time evolution of the theoretical temperature given by (1) is presented in figure 5 for $x=0$ (middle of the slip band) and for $x=1.3 \mathrm{~mm}$, location of point 2 . The maximum temperature increase which is measured at this location, $\Delta T_{\max }$, is $0.98^{\circ} \mathrm{C}$. Using the theoretical $T_{\max } / T_{0}$ the corresponding $\Delta T_{0}$ is evaluated to be approximately $140^{\circ} \mathrm{C}$ by . Of course, the thermogram of the point 1 cannot be used since its distance from the source which is very small is totally unknown due to the poor spatial resolution of the image.

The poor accuracy on the distance parameter is the weakness of the present method which also suffers a poor time resolution and a low signal to noise ratio.
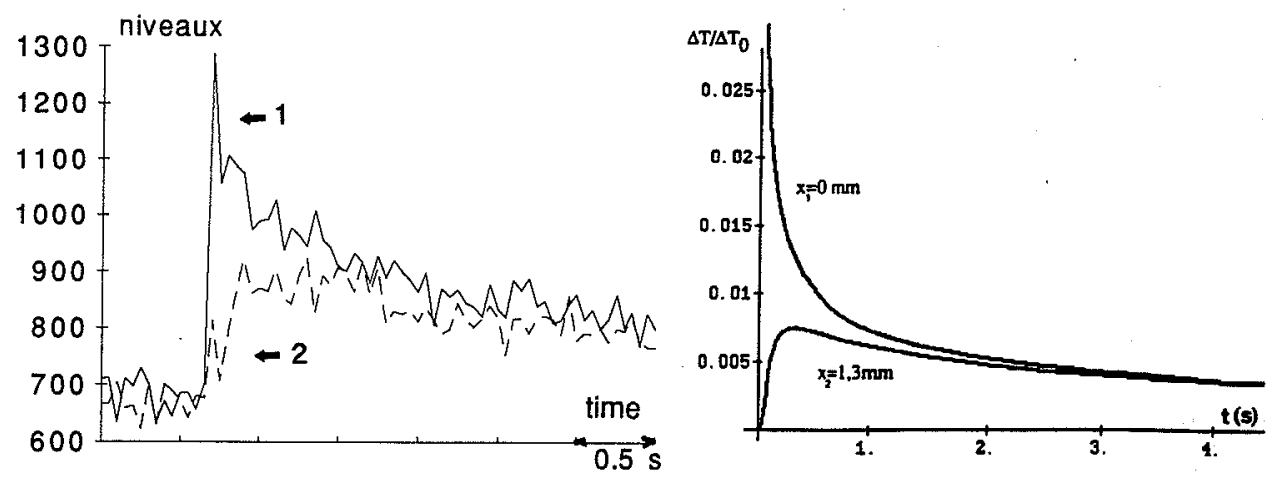

Fig. 4. - Experimental thermograms at very near of point1, the slip band,and at point point 2 located at $1,3 \mathrm{~mm}$ from it.

Fig. 5. - Theoretlcal thermograms at location $x=0$ (middle of the slip band) and at $x=1.3 \mathrm{~mm}$ (location of point 2) as given by eq. (1) 


\section{Calculation of the adiabatic initial heating}

These thermographic evaluations of the initial heating can be compared to a theoretical evaluation based on the tensile stress-strain curve observed during the instability. This curve presents drops occuring during the instabilities as shown in figure 6 . Assuming that the crosshead doesn't move during a drop (the specimen keeps constant total length, the plastic strain being balanced by an elastic compression) and, that the phenomenon is adiabatic, the following relation is obtained [3] :

$$
\Delta T_{0}=\alpha\left(V_{0} \sigma_{O} \Delta \sigma\right) /\left(M v_{0} C\right)
$$

where $V_{0}$ is the volume of the specimen, $\sigma_{0}$ the stress at which the stress drop occurs, $\Delta \sigma$ the amplitude of the stress drop, $M$ the combined elastic modulus of the specimen and of the testing machine, $v_{o}$ the slip band volume, $C$ the volume heat capacity, and $\alpha$ a constant generally assumed to be near of 0.9 (about $10 \%$ of the mechanical work being stored in the form of crystal defects). This formula leads to a $\Delta T_{0}$ value of $186^{\circ} \mathrm{C}$ in the case of the first event, analyzed using the 2-D scanning. This value must be considered as a maximum possible value (adiabatic assumption) which known with a poor accuracy, mainly due to the uncertainty related to the $\alpha$ parameter and the volume really active during the instability. The discrepancy from the thermographic evaluation is $20 \%$.
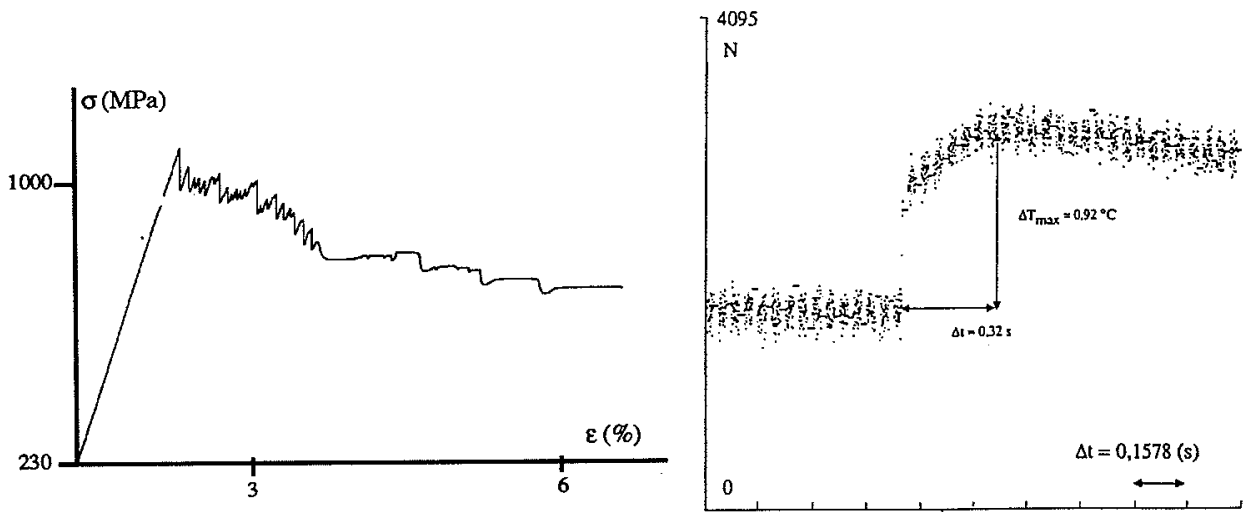

Fig. 6. - Stress-strain curve showing various instabilities occurred during the . test. Each event gives a vertical drop which allows an adiabatic estimation of the initlal heating.
Fig 7. -Thermogram corresponding to the point of maximum heating in the line this curve is a vertical line in the given in Figure $B$ ).

\section{Line scanning experiment}

A more precise determination of the initial heating $\Delta T_{0}$ is obtained using a line scanning. Practically this is obtained by stopping the motor of the line scanning system of the camera. Figure $B$ shows a part of the image obtained by this manner. The vertical axis is the time axis and the horizontal one the distance along a line, perpendicular to the specimen axis. The time delay between every two lines is $400 \mu \mathrm{s}$. The yellow and red upper part of the image corresponds to the initial temperature of the sample, near of the ambient. The heating appearing is seen as the blue zone which corresponds here to higher temperatures. The maximum heating measured is $0.92^{\circ} \mathrm{C}$. This value is relatively low, due to the fact the line is positioned at $1 \mathrm{~mm}$ from the slip band (a posteriori measurement). The width of this band was $40 \mu \mathrm{m}$. The corresponding $\Delta T_{0}$ value is $120^{\circ} \mathrm{C}$. The accuracy is better in the second experiment since the 


\section{http://dx.doi.org/10.21611/qirt.1992.020}

thermogram is defined by a higher number of digitized points (see figure. 7 ) than in the first (in fact 64 times more). In the present case the adiabatic evaluation based on the stress-strain curve leads to $180^{\circ} \mathrm{C}$, a value notably higher than the experimental one.

\section{Conclusion}

Infrared thermography was able to display the heating associated with plastic instabilities in the superalloy $A M 3$ in spite of the very short duration of such phenomenon.

The combination of the thermographic technique with a very simple heat diffusion model allows to experimentally estimate the initial temperature increase in the slip band. The experimental value of $140^{\circ} \mathrm{C}$ is not accurate, which is mainly due to the poor spatial resolution and the low signal/ratio of the image. A calculation based on mechanical analysis of the stressstrain curve can be compared, which leads to a value of $186{ }^{\circ} \mathrm{C}$. As the thermographic measurement, this last evaluation which is not accurate. The two values differ by $20 \%$, the mechanical evaluation being the higher, which is normal since it is based on adiabatic assumption.

An attempt to increase the accuracy of the thermographic measurement consisted in using a line-scanning procedure with the same camera. The temperature-time evolution at a given point of the specimen in this case is defined with a better time resolution ( 2500 points per second with a 36 points blanking every 100 points) allowing a more accurate evaluation of the initial heating in the slip band. In fact the horizontal scanning was not well adapted to the geometrical configuration of the mechanical setup and the uncertainty on the distance between the detection point and the slip band is yet present. Further tests are to be conducted with a vertical linescanning. With such configuration the time-evolution of the temperature in the slip band will be monitored giving a direct measurement of the initial heating. The use of more adapted optics (extension ring, extra lens) will ensure the spatial resolution needed.

\section{REFERENCES}

[1] T. KHAN (T.). - Recent developments and potentiel of single crystal superalloys for advanced turbine blades. High temperature alloys for gas turbines and other applications 86 , Part 1.edited by W. Betz et al., D. Reidel publishing Company, Dordrecht, 1986, p. 21-50.

[2] LISIECKI (B.), KUBIN (L.), and CARON (P.). - Tensile behaviour and plastic instabilities in single crystal AM3. Proc. Conf. ICSMA 9, Haifa (Israël), July 91, to be published.

[3] LISIECKI (B.), KUBIN (L.), CARON (P.) and LEVESQUE (P.) - Infrared thermography of plastic instabilities in superalloy single crystals - Proceedings of the 2nd European Conference on Advanced Materials and Processes (EUROMAT), Univ. of Cambridge, U.K., 22-24 July 1991, ed. T.W. Clyne and P.J. Withers, publ., The Institue of Materials, London-Brookfield, VCermont, 1992. 